\title{
High-Porosity Carbon Processed from Mixtures of Furfuryl Resin- Poly(ethylene glycol)-Benzenesulfonyl Chloride and Its Application for Catalyzing Tri-iodide Reduction
}

\author{
Shunjian $\mathrm{Xu}^{\mathrm{a}}$ \\ Xinyu Institute of New Energy, Xinyu University, Xinyu 338004, China
}

\begin{abstract}
High-porosity carbon (HPC) was first prepared from a novel mixture system of furfuryl resin as carbonyielding precursor, poly(ethylene glycol) with low molecular weight as pore-forming agent and benzenesulfonyl chloride (BSC) as cured catalyst, and then employed as catalyst for counter electrode in dye-sensitized solar cells (DSCs). The pore structures of HPC are tailored by changing BSC amount from $2 \%$ to $14 \%$. Especially, when BSC amount ascends from $4 \%$ to $8 \%$, the apparent porosity of HPC rises from $59.58 \%$ to $68.02 \%$ and the median pore size from 0.429 to $3.164 \mu \mathrm{m}$, except that some globular carbons initially occur on the carbon skeleton. Since the HPC derived from $4 \%$ BSC has high porosity and the most quantity of small-sized pores to enlarge its surface area allowing for the most catalytic active sites for tri-iodide reduction, the resulted counter electrode exhibits the best catalytic activity and the highest efficiency for use in DSCs.
\end{abstract}

\section{Introduction}

Porous carbons have been widely employed in many fields, such as energy storage, catalysis, purification and gas separation $[1,2]$. More recently the porous carbon has been developed as an alternative catalyst to replace the platinum for counter electrode in dye-sensitized solar cells (DSCs), due to its high corrosion inert, low cost and admirable catalytic activity $[3,4]$. In such application, the pore structure of porous carbon, such as apparent porosity, surface area and pore size, is very critical in determining the catalytic activity of the electrode for the tri-iodide $\left(\mathrm{I}_{3}^{-}\right)$ reduction and the final photovoltaic performances of the device.

The polymerization-induced phase separation and pyrolysis (PIPSP) is an impactful route to tailor the pore structure of porous carbon [5-7]. In this process, carbonyielding precursor (CYP) is first mixed with poreforming agent (PFA) and optional additives (such as starch and water). The polymerization of CYP results in the phase separation and a rigid body. The rigid body is then pyrolyzed to form porous carbon with removing fluid PFA. The pore structure of the porous carbon can be controlled by changing the composition of CYP-PFA system. Therefore, to understand the relationship between the composition of CYP-PFA system and the pore structure of the porous carbon is indispensable for the modification and application of the porous carbon. To date, many CYP-PFA systems, such as phenol resin (PF)ethylene glycol (EG) [5], poly(furfuryl alcohol)polyethylene glycol (PEG) with large molecular weight [6] and (PF+furfuryl alcohol)-EG [7], have been

\footnotetext{
a Corresponding author: xushunjian@126.com
}

developed to fabricate the porous carbons. However, few reports have addressed the potential synthesis of the porous carbon based on a system of furfuryl resin (FR), PEG with low molecular weight and benzenesulfonyl chloride (BSC) as well as the application of porous carbon with high porosity as catalyst in DSCs.

In this study, the novel system containing FR, lowmolecular-weight PEG and BSC was first developed to fabricate high-porosity carbon (HPC), with emphasis on controlling the pore structure of the HPC through changing BSC amount. The maximum apparent porosity of the HPC reaches $68.02 \%$. The HPC was then employed as catalyst for counter electrode in DSCs.

\section{Experimental}

\subsection{Preparation and characterizations of porous carbon}

To synthesize the HPC, FR (Industrial level), PEG (CP, Average molecular weight: 190-210), BSC (CP, 98.5\%) and deionized water were mixed by stirring mechanically at room temperature, then poured into mold and cured at $40{ }^{\circ} \mathrm{C}$ for $3 \mathrm{~h}$. The sample was then postcured at $100{ }^{\circ} \mathrm{C}$ for $40 \mathrm{~h}$. After $40 \mathrm{~h}$, the rigid body was removed from the mold. The sample was finally pyrolyzed by slowly ramping to $800{ }^{\circ} \mathrm{C}$ in a flowing $\mathrm{N}_{2}$, followed by cooling naturally to room temperature. The weight ratio of FR and PEG was $2: 3$. The weight fraction of BSC was $2 \%$, $4 \%, 8 \%$ and $14 \%$, respectively. And the resulting HPC 
was designated as HPC-1, HPC-2, HPC-3 and HPC-4, accordingly.

The crystal structures of HPC were identified by $\mathrm{X}$-ray diffraction (XRD; D8 ADVANCE, Bruker). The morphologies of HPC were characterized by scanning electron microscopy (SEM; EVO MA10, Zeiss). The median pore size, apparent porosity, bulk density and pore size distributions of HPC were characterized by mercury porosimetry (Autopore 9510 , Micromeritics).

\subsection{Configuration and measurements of porous carbon based cells}

The HPC counter electrode was prepared by coating a blended paste on FTO, and then heated to $120^{\circ} \mathrm{C}$ for $2 \mathrm{~h}$. The blended paste was obtained by grinding $150 \mathrm{mg} \mathrm{HPC}$ powder, $15 \mathrm{mg}$ carboxymethyl cellulose (CMC) and 2.5 $\mathrm{ml}$ alcohol aqueous solution. The DSCs was assembled with a N719 dye-sensitized $\mathrm{TiO}_{2}$ photoanode and a HPC counter electrode sandwiching an electrolyte. The electrolyte was a blended solvent of acetonitrile and EG $\begin{array}{llllllllll}\text { containing } & 0.5 & \mathrm{M} & \mathrm{KI} & 0.05 & \mathrm{M}^{2} & \mathrm{I}_{2} & \text { and } & 0.5 & \mathrm{M}\end{array}$ tributylphosphate. The photoanode was prepared as following [3]: $\mathrm{TiO}_{2}$ film was firstly coated on FTO substrate by printing a paste containing anatase $\mathrm{TiO}_{2}$ particles. After sintering at $450{ }^{\circ} \mathrm{C}$ for $30 \mathrm{~min}$, the film was treated with $40 \mathrm{mM} \mathrm{TiCl}_{4}$ aqueous solution, and then further heat-treated at $450{ }^{\circ} \mathrm{C}$ for $30 \mathrm{~min}$. The photoanode was finally obtained by immersing the $\mathrm{TiO}_{2}$ film into a N719 ethanol solution $(0.5 \mathrm{mM})$ at room temperature for $24 \mathrm{~h}$.

Cyclic voltammetry (CV) experiments were performed using a three-electrode system. Electrochemical impedance spectroscopy (EIS) measurements were conducted at $10 \mathrm{mV}$ of amplitude. Photocurrent-voltage $(J-V)$ curves of DSCs were measured under the illumination of a Xenon light source (CHF-XM500, Trusttech). The light intensity was 100 $\mathrm{mW} \cdot \mathrm{cm}^{-2}$.

\section{Results and discussion}

Fig. 1 illustrates the micrographs of HPC-1 to HPC-4. It is obvious that the morphologies of HPC show differences. Both HPC-1 and HPC-2 have interconnected porous structure with smooth carbon skeleton, and the carbon skeleton in HPC-2 is slightly finer than that in HPC-1. In contrast, with the increase of BSC amount to $8 \%$ or $14 \%$ (HPC-3 or HPC-4), some globular carbons stacked on the carbon skeleton are detected, except that the carbon skeleton becomes coarse. Accompanied by the evident differences of carbon skeletons, the pore characteristics also vary obviously, which can be confirmed by the pore size distributions and pore characteristic parameters of HPC, as shown in Fig. 2(a) and Table 1, respectively. From HPC-2 to HPC-3, the range of main pore size distribution changes from $0.2 \sim$ $0.6 \mu \mathrm{m}$ to $2 \sim 4 \mu \mathrm{m}$, and the median pore size $\left(\Phi_{\mathrm{m}}\right)$ ascents from 0.429 to $3.164 \mu \mathrm{m}$. Furthermore, the apparent porosity $\left(P_{\mathrm{a}}\right)$ rises from $59.58 \%$ to $68.02 \%$. Table 1 also displays that the $\Phi_{\mathrm{m}}$ of HPC-2 is slightly smaller than that of HPC-1, and the $P_{\mathrm{a}}$ of both HPC are almost identical, indicating higher surface area for HPC-2. In addition, the bulk density $\left(\rho_{\mathrm{b}}\right)$ of HPC decreases from 0.601 to $0.472 \mathrm{~g} \cdot \mathrm{cm}^{-3}$ with increasing BSC amount.

The images of rigid body reveal that the morphology of HPC is inherited from that of the rigid body, as shown in the inserts in Figs. 1(b) and (c). The pyrolysis mainly brings about the contraction of the pore size and wall thickness. It indicates that the different morphologies of HPC with various BSC amount are formed during curing.

The XRD patterns of HPC are demonstrated in Fig. 2(b). The position and intensity of both (002) and (100) peaks are almost unchanged with increasing BSC amount, suggesting similar crystallinity for all HPC [8].The influence of BSC amount on the gel time of FR is demonstrated in Fig. 2(c). It is obvious that the gel time falls rapidly with increasing the BSC amount from $2 \%$ to $8 \%$, and then declines slightly with further increasing the BSC amount.
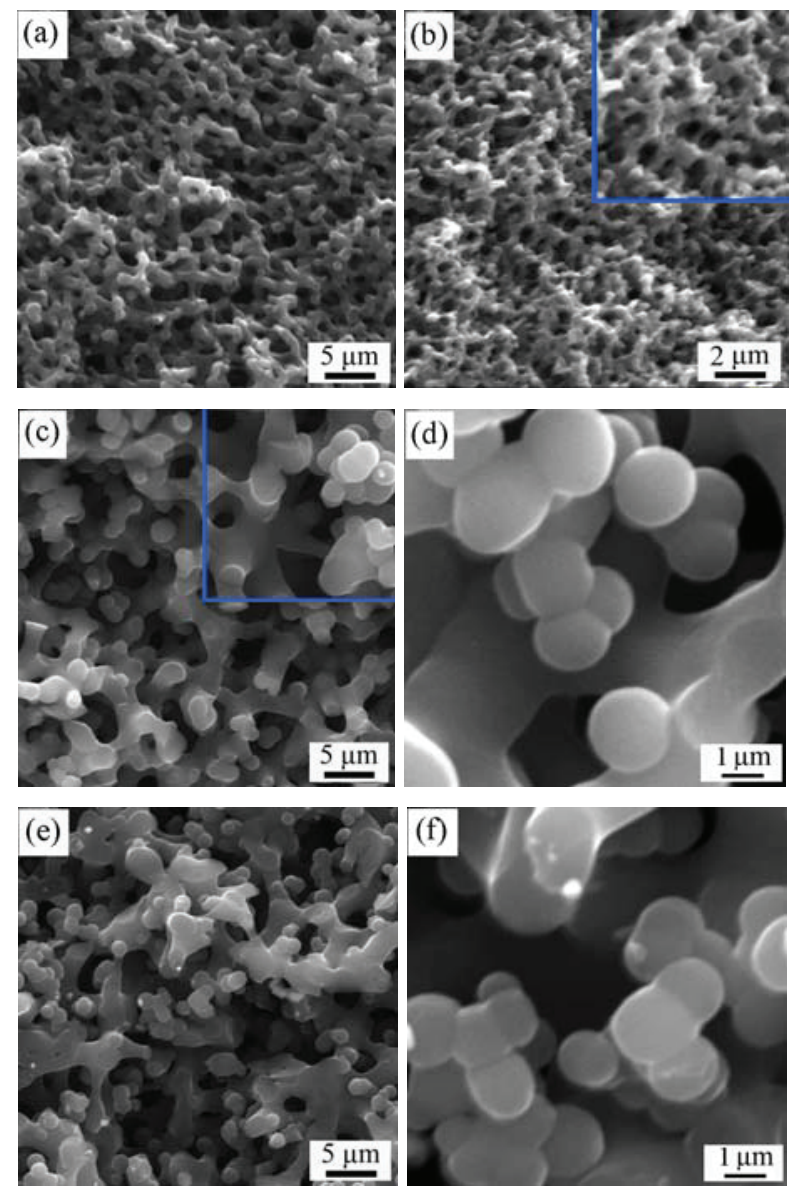

Figure 1. Variation in morphologies of (a) HPC-1; (b) HPC-2; (c) and (d) HPC-3; (e) and (f) HPC-4. The inserts show the images of the corresponding rigid bodies (before pyrolysis).

The formation and different morphologies of the HPC should be preliminarily explained as follows. The BSC as a cured catalyst accelerates FR polymerization (Fig. 2c) and further changes the phase separation kinetics in FRPEG-BSC system during curing, resulting in the patterned variation of FR-rich and PEG-rich phases in rigid body. After pyrolysis, the HPC inherits the morphologies of rigid body with the transformation of 
FR-rich phase into carbon skeleton and the remove of PEG-rich phase to form pores (Figs. 1 and 2b). Under high BSC amount (reached $8 \%$ or $14 \%$ ), the fast polymerization of FR promotes the growing-up of FRrich and EG-rich phases. Meanwhile, the rapid growing- up of EG-rich phase would interrupts the interconnection of partial FR-rich phase, and the opened FR-rich phase tends to form globularity under surface tension. Therefore, the HPC (HPC-3 or HPC-4) has large pores and coarse carbon skeleton, except that some globular carbons occur.
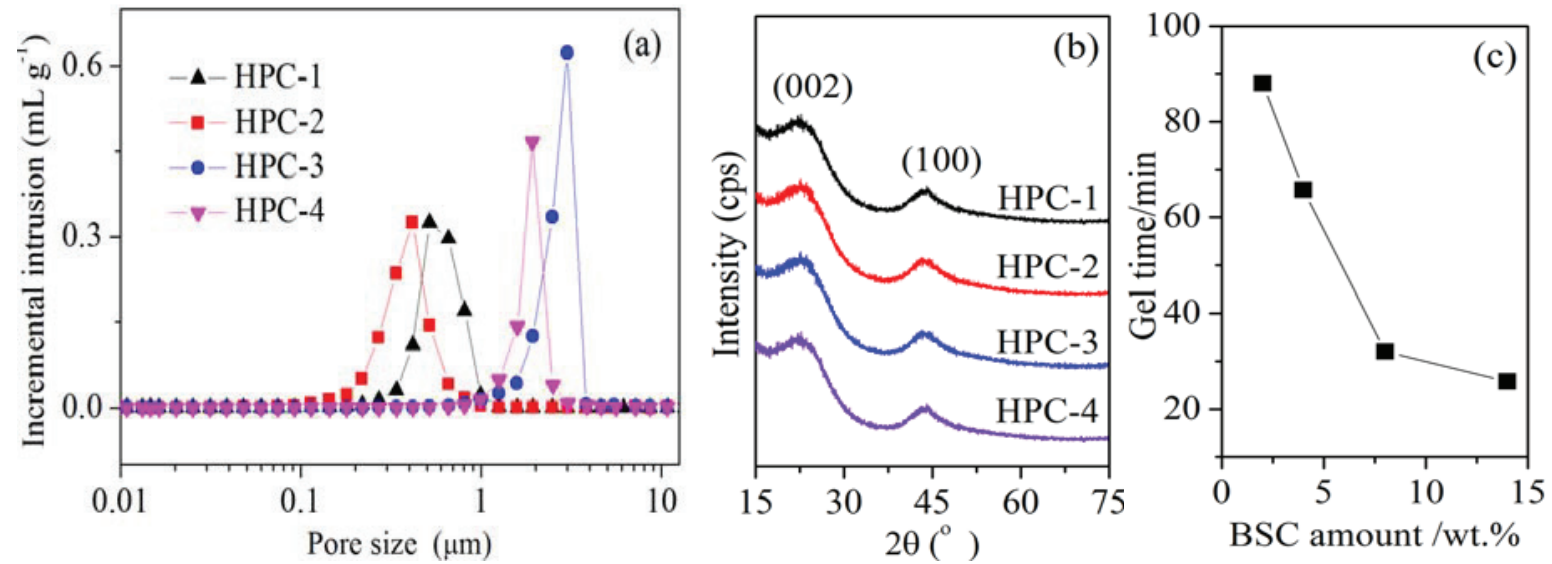

Figure 2. (a) Pore size distributions of HPC-1 to HPC-4; (b) XRD patterns of HPC-1 to HPC-4; (c) Relationship between the BSC amount and the gel time of FR.

Table 1. Pore characteristic parameters of HPC and their photovoltaic performances for use in DSCs.

\begin{tabular}{llllllll}
\hline Catalyst & $\begin{array}{l}\Phi_{\mathrm{m}} \\
(\mu \mathrm{m})\end{array}$ & $\begin{array}{l}P_{\mathrm{a}} \\
(\%)\end{array}$ & $\begin{array}{l}\rho_{\mathrm{b}} \\
\left(\mathrm{g} \cdot \mathrm{cm}^{-3}\right)\end{array}$ & $\begin{array}{l}J_{\mathrm{sc}} \\
\left(\mathrm{mA} \cdot \mathrm{cm}^{-2}\right)\end{array}$ & $\begin{array}{l}V_{\mathrm{oc}} \\
(\mathrm{V})\end{array}$ & $F F$ & $\begin{array}{l}\eta \\
(\%)\end{array}$ \\
\hline HPC-1 & 0.658 & 59.82 & 0.601 & 11.58 & 0.676 & 0.385 & 3.01 \\
HPC-2 & 0.429 & 59.58 & 0.593 & 12.57 & 0.690 & 0.461 & 4.00 \\
HPC-3 & 3.164 & 68.02 & 0.473 & 11.85 & 0.672 & 0.357 & 2.84 \\
HPC-4 & 2.601 & 63.70 & 0.472 & 8.63 & 0.696 & 0.336 & 2.02 \\
\hline
\end{tabular}

${ }^{*} \Phi_{\mathrm{m}}$ : Median pore size; $P_{\mathrm{a}}$ : Apparent porosity; $\rho_{\mathrm{b}}$ : Bulk density; $J_{\mathrm{sc}}$ : Short-circuit current density; $V_{\mathrm{oc}}$ : Open-circuit voltage; $F F$ : Fill factor; $\eta$ : Efficiency.
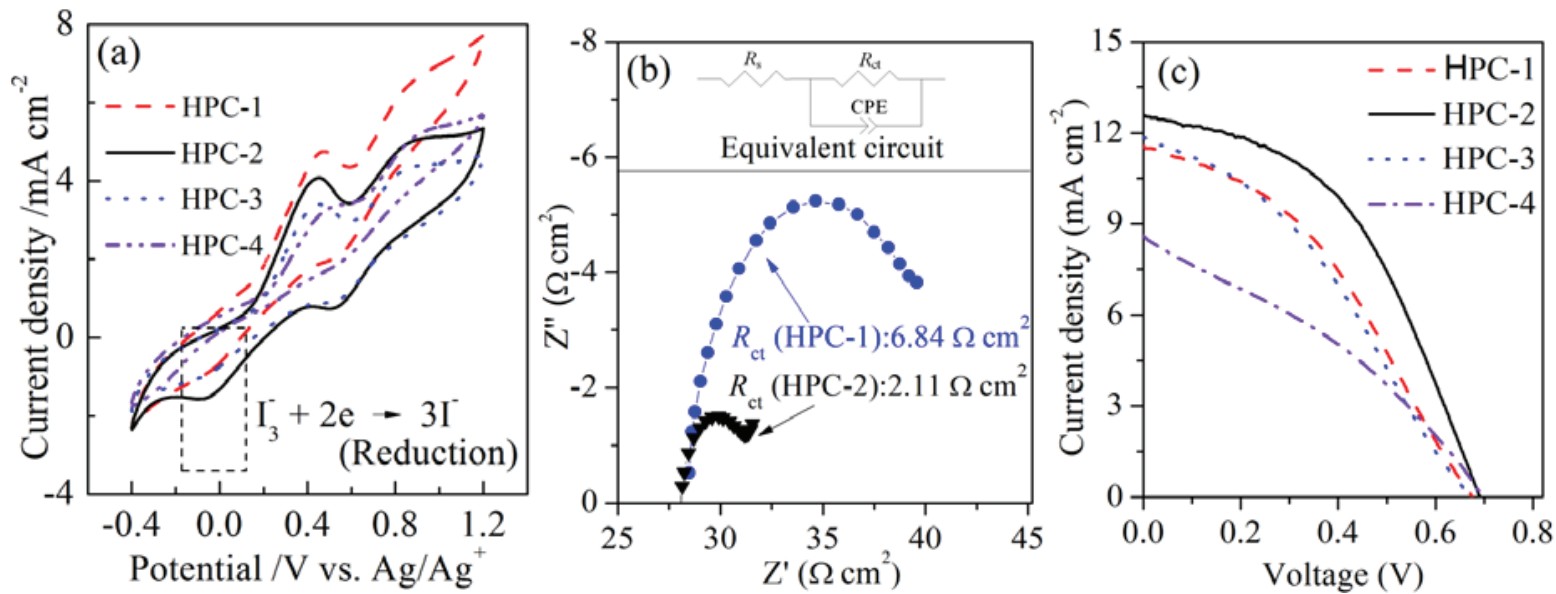

Figure 3. Catalytic activities of HPC and their photovoltaic performances for use in DSCs. (a) CVs of HPC as counter electrode; (b) EIS of HPC as counter electrode; (c) $J-V$ curves of HPC based DSCs.

The CV curves of HPC as counter electrode are shown in Fig. 3(a). In the peak represented the reduction of $\mathrm{I}_{3}^{-}$to $\mathrm{I}^{-}$, the current density of HPC-2 is higher than that of HPC-1 or HPC-3, and is far higher than that of HPC-4. It suggests that HPC-2 has the best catalytic activity [9, 10].The EIS displayed in Fig. 3(b) confirms that the catalytic activity of HPC-2 is better than that of HPC-1. The values of charge transfer resistance $\left(\mathrm{R}_{\mathrm{ct}}\right)$ are 2.11 and $6.84 \Omega \mathrm{cm}^{2}$ corresponding to HPC-2 and HPC-1, respectively. The excellent catalytic activity of HPC-2 is mainly attributed to its distinctive pore structure since the difference of crystal structure among HPC can be ignorable. Compared with HPC-1, HPC-3 or HPC-4, HPC-2 possesses more pores with small size to enlarge its surface area allowing for more catalytic active sites for the $\mathrm{I}_{3}{ }^{-}$reduction. As a result, $\mathrm{HPC}-2$ has better catalytic activity. The $J-V$ curves of HPC based DSCs are illustrated in Fig. 3(c). The high catalytic activity of HPC brings about the high efficiency $(\eta)$ for use in DSCs. The 
$\eta$ of DSCs with HPC-2, HPC-1, HPC-3 and HPC-4 is 4\%, $3.01 \%, 2.84 \%$ and $2.02 \%$, respectively, as shown in Table 1.

For comparison, two conventional carbon materials, graphite and activated carbon (AC), were individually used as catalysts to prepare the counter electrodes, and the $\eta$ values of the corresponding DSCs were $0.96 \%$ and $2.71 \%$, respectively [11]. It is clear that the HPC-1, HPC2 or HPC-3 is superior to the graphite or $\mathrm{AC}$ for catalyzing the $\mathrm{I}_{3}^{-}$reduction.

\section{Summary}

HPC is fabricated from a novel FR-PEG-BSC system, and its pore structures are tailored by changing $\mathrm{BSC}$ amount. Especially, when BSC amount ascends from 4\% to $8 \%$, the apparent porosity of HPC rises from $59.58 \%$ to $68.02 \%$ and the median pore size from 0.429 to $3.164 \mu \mathrm{m}$, except that some globular carbons initially occur on carbon skeleton. The CV and EIS show that HPC derived from 4\% BSC as counter electrode exhibits the best catalytic activity, which is mainly attributed to the enough catalytic active sites on its large skeleton surface. Importantly, the high catalytic activity of HPC brings about the high efficiency for use in DSCs.

\section{Acknowledgements}

This work was financially supported by National Natural Science Foundation of China (51462035), Training Programme Foundation for Young Scientist of Jiangxi (20133BCB23035), Natural Science Foundation of Jiangxi (20161BAB206106) and Educational Commission of Jiangxi (KJLD13100).

\section{References}

1. Z. Li, W. Yang, X.W. Xu, Y.S. Tang, Z.W. Zeng, F. Yang, L.Q. Zhang, G.Q. Ning, C.M. Xu, Y.F. Li, J. Power Sources 327, 289 (2016)

2. H. Hadoun, Z. Sadaoui, N. Souami, D. Sahel, I. Toumert, Appl. Surf. Sci. 280, 1 (2013)

3. S.J. Xu, G.W. Liu, J. Li, G.J. Qiao, Electrochim. Acta 76, 504 (2012)

4. S.Q. Fan, B. Fang, J.H. Kim, B. Jeong, C. Kim, J.S. Yu, J.J. Co, Langmuir 26, 13644 (2010)

5. S.J. Xu, J. Li, G.J. Qiao, H.J. Wang, T.J. Lu, Carbon 47, 2103 (2009)

6. M.S. Strano, H. Agarwal, J. Pedrick, D. Redman, H.C. Foley, Carbon 41, 2501 (2003)

7. Y. Zhang, Z. Yuan, Y. Zhou, Mater. Lett. 109, 124 (2013)

8. A. Braun, M. Bärtsch, B. Schnyder, R. Kötz, O. Haas, H.G. Haubold, G. Goerigk, J. Non-Cryst. Solids 260, 1 (1999)

9. W.Y. Cheng, C.C. Wang, S.Y. Lu, Carbon 54, 291 (2013)

10. G.T. Yue, J.H. Wu, Y.M. Xiao, M.L. Huang, J.M. Lin, L.Q. Fan, Z. Lan, Electrochim. Acta 92, 64 (2013)
11. Y.F. Luo, X.Y. Liu, S.J. Xu, Z. Wei, New Chem. Mater 40, 58 (2012) 